higher as a consequence as community pharmacists, unlike their hospital based colleagues, cannot usually buy in bulk when they are only rarely dispensing certain drugs. Nor do they have the preferential pricing advantage offered to their hospital colleagues. The motivation to shift prescribing costs from hospitals to general practice would be reduced if the relevant health authorities - that is, the district health authorities and the family health services authorities held a common drug budget or if virement between drug and other budgets in hospitals was disallowed.

There are also possible adverse implications for patient care. General practitioners may lack the knowledge and, in some cases, the technical resources needed to monitor the dosage, side effects, and response of patients to specialist hospital drug regimens. They do not have access to the skill of the ward pharmacist, a service available to the hospital doctor. Patients may find it difficult to arrange at short notice for their general practitioner to continue their hospital initiated drug treatments. In addition, they may find that they have to pay for two prescriptions when one is sufficient.

There are also possible advantages. The savings made by hospitals in their drug budgets could be used to increase the numbers of patients treated and the range of services offered. Transferring prescribing responsibility to general practitioners may reduce the confusion which can arise from having two prescribers (that is, the general practitioner and the consultant) for one patient and should help to clarify which doctor bears the clinical responsibility. Patients may prefer to obtain their prescription from the community pharmacist for reasons of accessibility, the convenience, hours of opening, and availability of non-pharmaceutical products.

The current trend towards more restrictive outpatient dispensing may have both beneficial and negative effects on the quality of outpatient care. In subsequent papers we examine the opinions of general practitioners, hospital consultants, and hospital and community pharmacists about the implications of current hospital outpatient dispensing policies.

We thank particularly the chief pharmacists of all the hospitals who participated in the study, and Linda MacraeSamuel, Frances Smyth, and Mary Taylor for their valuable help with research. The study was funded by a grant from the Department of Health.

1 Economics of prescribing. Lancet 1989;i:981.

2 Beecham L. Transferring prescribing costs. BMF 1989;299:863.

3 Kately GD. Comparative costs of supplying medicines to outpatients - a study of in-house dispensing. V. The use of FPlO(HP) forms. Pharmacy Manageof in-house dispens.
ment. 1987;13:5-7.

4 Department of Health. Improving prescribing. The implementation of the GP indicative prescribing scheme. London: HMSO, May 1990.

(Accepted 25 September 1991)

\title{
Prescribing at the hospital-general practice interface. II: Impact of hospital outpatient dispensing policies in England on general practitioners and hospital consultants
}

\author{
Bonnie Sibbald, Patricia Wilkie, James Raftery, Stuart Anderson, Paul Freeling
}

\section{Abstract}

Objective-To assess the impact on general practitioners and hospital consultants of hospital outpatient dispensing policies in England.

Design-Postal questionnaire and telephone interview survey of general practitioners and hospital consultants in January 1991.

Setting-94 selected major acute hospitals in England.

Participants-20 general practitioners in the vicinity of each of 94 selected hospitals and eight consultants from each, selected by chief pharmacists.

Main outcome measures-Proportions of general practitioners unable to assume responsibility for specialist drugs and of consultants wishing to retain responsibility; association between dispensing restrictions and the frequency of general practitioners being asked to prescribe hospital initiated treatments.

Results-Completed questionnaires were obtained from 1207 (64\%) of 1887 general practitioners and $457(63 \%)$ of 729 consultants. $570(46 \%)$ general practitioners felt unable to take responsibility for certain treatments, principally because of difficulty in detecting side effects $(367,30 \%)$, uncertainty about explaining treatment to patients $(332,28 \%)$, and difficulty monitoring dosage (294, $24 \%)$. Among consultants $328(72 \%)$ wished to retain responsibility, principally because of specialist need for monitoring $(93,20 \%)$, urgent need to commence treatment $(64,14 \%)$, and specialist need to initiate or stabilise treatment $(63,14 \%)$. The more restricted the drug supply to outpatients, the more frequently consultants asked general practitioners to prescribe $(p<0.01)$ and complete a short course of treatment initiated by the hospital $(p<0.001)$.

Conclusions-Restrictive hospital outpatient dispensing shifts clinical responsibility on to general practitioners. Hospital doctors should be able to retain responsibility for prescribing when the general practitioner is unfamiliar with the drug or there is a specialist need to initiate, stabilise, or monitor treatment.

\section{Introduction}

The two level health care system in Britain relies on general practitioners referring patients to hospital based specialists who advise on appropriate treatment and, when necessary, undertake treatment. The decision as to which doctor is best able to assume clinical responsibility, and therefore responsibility for prescribing, should be negotiated between the individuals concerned. Recently, however, concern has been expressed that prescribing at the hospitalgeneral practice interface may have become governed by considerations of cost and available resources rather than professional considerations. ${ }^{1-4}$

Cash limited hospitals can save funds by shifting outpatient prescribing costs on to general practitioners. The concern expressed by general practitioners is likely to be increased now that there are indicative prescribing allowances in general practice and even tighter controls on hospital budgets through contracts. ${ }^{56}$ In an accompanying paper we suggested that the supply of drugs given to outpatients was being further restricted. ${ }^{7}$ The impact on patient care may be 
adverse if general practitioners lack the necessary knowledge or resources to prescribe specialist hospital drugs.

No systematic investigation of these problems of prescribing at the hospital-general practice interface has been reported. We therefore surveyed the opinions of general practitioners and consultants in England about the impact of current hospital outpatient dispensing policies on their work.

\section{Methods}

A sample of six to eight hospitals in each region was selected by hand from those who had responded to our questionnaire on current outpatient dispensing policy.' Each sample $(a)$ included at least one teaching hospital in addition to district general hospitals; $(b)$ represented the range in the limits imposed by hospitals on the quantities of drugs supplied to outpatients within the region; and $(c)$ gave the widest possible geographical spread. The numbers included for each region varied because in some regions closure of pharmacies and a low (or late) response to the initial survey meant that there were insufficient numbers from which to draw the sample.

We used family health services authorities' lists to identify 20 general practitioners in the vicinity of each selected hospital and selected one general practitioner from each practice. In some instances there were insufficient practices to meet the target number. In January 1991, 1873 general practitioners so identified were sent a questionnaire about the impact on them of their hospital's outpatient dispensing policy. We believed that a repeat posting would not increase response and therefore administered an identical questionnaire to non-responders by telephone.

Consultants were drawn from eight medical special-

TABLE I-Problems relating to clinical responsibility among 1207 general practitioners asked by hospital to prescribe drugs ${ }^{\star}$

\begin{tabular}{lc}
\hline & No $(\%)$ \\
\hline Unable to take clinical responsibility for: & \\
Fertility drugs & $242(20)$ \\
Growth hormone & $86(7)$ \\
Immunosuppressants & $72(6)$ \\
Other hormones & $58(5)$ \\
Cytotoxic drugs & $54(4)$ \\
Erythropoietin & $53(4)$ \\
Continuous ambulatory peritoneal dialysis fluid & $38(3)$ \\
Cardiovascular drugs & $29(2)$ \\
Reasons why unable to take responsibility: & $367(30)$ \\
Detecting side effects & $332(28)$ \\
Explaining treatment to patient & $294(24)$ \\
Monitoring dosage & $178(15)$ \\
Cost & $116(10)$ \\
Lack of knowledge & \\
Suggestions for clarifying clinical responsibility: & \\
Prompt communication to general practitioner from & $901(75)$ \\
$\quad$ consultant, describing course of treatment & \\
Referral letter from general practitioner to consultant states & \\
$\quad$ whether consultant is to treat or only to advise & $497(41)$ \\
Patient held form notifies general practitioner of consultant's & \\
$\quad$ diagnosis and proposed treatment & $422(35)$ \\
\hline
\end{tabular}

* Some general practitioners gave more than one response and other declined to answer all questions.

TABLE II - Impact of hospital dispensing policy on general practitioner and consultant prescribing. Figures are numbers (percentages) of doctors

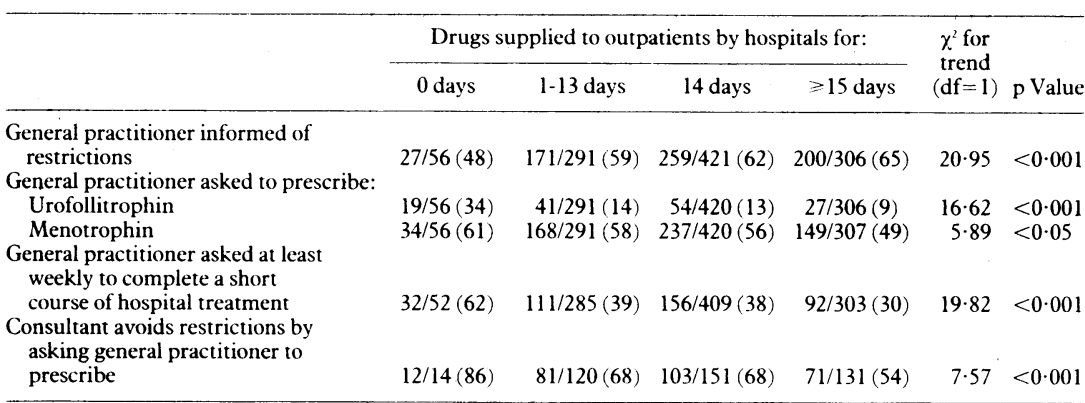

ties likely to experience prescribing problems at the interface with general practice: cardiology, gastroenterology, respiratory medicine, rheumatology, paediatrics, renal medicine, dermatology, and gynaecology. In January 1991 the chief pharmacist in each selected hospital was asked to distribute a questionnaire with prepaid reply envelope to one consultant in each specialty. When there was no consultant in a designated specialty the questionnaire was sent to a consultant in general medicine with a particular interest in that specialty. Questionnaires addressed the impact on consultants of the hospital's outpatient dispensing policy. We were not notified of consultants' names and thus could not follow up non-responders.

Data analysis - Data were coded and analysed with the statistical package for the social sciences (SPSS/ $\mathrm{PC}+$ ). Information collected from the previous survey of hospital outpatient dispensing policies ${ }^{7}$ was merged with that from the current survey to examine relations between a hospital's dispensing policy and the views of the doctors working in or near it. The significance of the association between variables was assessed with the $\chi^{2}$ test or $\chi^{2}$ test for trend, as appropriate.

\section{Results}

\section{GENERAL PRACTITIONERS}

Completed questionnaires were obtained from 1207 $(64 \%)$ of the 1873 general practitioners approached. Responders were drawn from 58 of the 90 family health services authorities in England. Among the nonresponders, many declined to participate because of pressure of work. A few of those approached by telephone refused to participate because we were unable to pay them.

We compared the responses in the postal questionnaires with those in the telephone interviews to assess the possible bias introduced by this change in data collection. There was good agreement for the proportions of doctors who had been asked to prescribe specialist hospital treatments, the proportions who had objected to this, and the nature of their objections. We concluded that no major bias had been introduced, and the final analysis was carried out on the combined data.

Overall, $570(46 \%)$ general practitioners said that they had been asked by the hospital to prescribe drugs for which they felt unable to take clinical responsibility, such as fertility treatments, growth hormone, anticancer treatments (immunosuppressants and cytotoxic drugs), and drugs used in renal failure (fluid for continuous ambulatory peritoneal dialysis and erythropoietin). When general practitioners were asked why they felt unable to take clinical responsibility for these drugs the principal reasons they endorsed from a list of possibilities were difficulty in detecting side effects, uncertainty about explaining treatment to patients, difficulty monitoring dosage, high cost of drug, and lack of knowledge about the treatment (table I). An additional problem was general practitioners being asked to complete a short course of drug treatment initiated by the hospital. Such requests were received weekly by 428 general practitioners, monthly by 428 , and yearly by 172 .

When general practitioners were asked what procedures might help to clarify who had clinical responsibility for outpatients the procedures they most commonly endorsed from a list of possibilities were prompt communication from the consultant, describing the suggested course of treatment; clarification in the general practitioner's letter of referral whether the consultant should treat or only advise about treatment; and patient held forms notifying the general practitioner of the consultant's diagnosis and proposed treatment (table I).

A total of $1069(89 \%)$ general practitioners said that 


\begin{tabular}{lcc}
\hline Specialty & $\begin{array}{c}\text { No } \\
\text { approached }\end{array}$ & $\begin{array}{c}\text { No }(\%) \\
\text { completing } \\
\text { form }\end{array}$ \\
\hline Cardiology & 91 & $53(58)$ \\
Dermatology & 91 & $51(56)$ \\
Gastroenterology & 91 & $58(64)$ \\
Gynaecology & 91 & $48(53)$ \\
Paediatrics & 91 & $71(78)$ \\
Renal medicine & 91 & $56(62)$ \\
Respiratory medicine & 91 & $52(57)$ \\
Rheumatology & 91 & $67(74)$ \\
\hline Total & 728 & $457(63)$ \\
\hline
\end{tabular}

TABLE IV-Problems relating to clinical responsibility among 457 consultants ${ }^{\star}$

\begin{tabular}{ll}
\hline & No(\%) \\
\hline $\begin{array}{l}\text { Circumstances under which responsibility for prescribing } \\
\text { should be retained: }\end{array}$ & \\
Specialist needed to monitor drug & $93(20)$ \\
Patient condition acute or complex & $64(14)$ \\
Specialist needed to initiate or stabilise treatment, or both & $63(14)$ \\
Non-compliance by general practitioner & $23(5)$ \\
Drugs for which responsibility for prescribing should be retained: & $65(14)$ \\
Cytotoxic drugs & $33(7)$ \\
Immunosuppressants & $18(4)$ \\
Second line antirheumatic drugs & $14(3)$ \\
Antituberculosis drugs & $12(3)$ \\
Fertility drugs & \\
Suggestions to clarify clinical responsibility: & $379(83)$ \\
Prompt communication to general practitioner from & \\
$\quad$ consultant describing course of treatment & \\
Referral letter from general practitioner to consultant states if & \\
latter to treat or only advise & $203(44)$ \\
Patient held form notifies general practitioner of consultant's & $177(39)$ \\
$\quad$ diagnosis and proposed treatment & \\
\hline
\end{tabular}

* Some consultants gave more than one response and others declined to answer all questions.

TABLE $\mathrm{V}-E$ ffect of restrictions on outpatient dispensing on consultants ${ }^{\star}$

\begin{tabular}{|c|c|c|c|c|}
\hline \multirow[b]{2}{*}{ Specialty } & \multirow[b]{2}{*}{$\begin{array}{l}\text { Total } \\
\text { No }\end{array}$} & \multicolumn{3}{|c|}{ No $(\%)$ consultants: } \\
\hline & & $\begin{array}{l}\text { Needing to } \\
\text { breach restrictions } \\
\text { weekly }\end{array}$ & $\begin{array}{l}\text { Avoiding restrictions } \\
\text { by asking } \\
\text { general practitioner } \\
\text { to prescribe }\end{array}$ & $\begin{array}{c}\text { Asking general } \\
\text { practitioner to } \\
\text { complete short } \\
\text { treatment course at } \\
\text { least weekly }\end{array}$ \\
\hline Cardiology & 52 & $9(17)$ & $27(52)$ & $12(23)$ \\
\hline Dermatology & 51 & $21(41)$ & $31(61)$ & $23(45)$ \\
\hline Gastroenterology & 58 & $10(17)$ & $39(67)$ & $29(50)$ \\
\hline Gynaecology & 48 & $6(13)$ & $37(77)$ & $33(69)$ \\
\hline Paediatrics & 71 & $12(17)$ & $45(63)$ & $19(27)$ \\
\hline Renal medicine & 55 & $9(16)$ & $39(71)$ & $18(33)$ \\
\hline Respiratory medicine & 52 & $15(29)$ & $22(42)$ & $16(31)$ \\
\hline Rheumatology & 68 & $10(15)$ & $46(68)$ & $21(31)$ \\
\hline Total & 455 & $92(20)$ & $286(63)$ & $171(38)$ \\
\hline $\begin{array}{l}\chi^{2} \\
\text { Degrees of freedom } \\
\text { Significance }\end{array}$ & & $\begin{array}{l}39 \cdot 3 \\
21 \\
\mathrm{p}<0 \cdot 01\end{array}$ & $\begin{array}{c}19 \cdot 3 \\
7 \\
\mathrm{p}<0 \cdot 01\end{array}$ & $\begin{array}{c}52.8 \\
21 \\
\mathrm{p}<0 \cdot 001\end{array}$ \\
\hline
\end{tabular}

^Some consultants declined to answer all questions.

the hospitals to which they referred patients restricted the time for which outpatient prescriptions could be given. The more restrictive the hospital's policy the less likely the general practitioner was to have been informed before its introduction; the more likely he or she was to have been asked to prescribe fertility drugs (urofollitrophin and menotrophin); and the more likely he or she was to have been asked to complete a short course of treatment initiated by the hospital (table II).

\section{CONSULTANTS}

Three of the 94 selected hospitals declined to distribute questionnaires to consultants. Completed questionnaires were obtained from $457(63 \%)$ of the 728 consultants approached in the 91 participating hospitals. Responders were drawn from 91 of the 190 district health authorities in England. Table III summarises the response rate by specialty.

Overall, $328(72 \%)$ consultants said that there were circumstances in which they wished to retain clinical responsibility, including responsibility for prescribing (table IV). The circumstances most often volunteered were specialist needed to initiate or stabilise drug treatment, or both; specialist needed to monitor drug treatment; patients with complex or acute conditions; and unlikelihood of general practitioner complying with recommendations (table IV). The reasons given for retaining clinical responsibility did not differ appreciably among the specialties.

A total of $357(78 \%)$ consultants passed responsibility for prescribing to the general practitioner while retaining responsibility for monitoring. Consultant gastroenterologists were the most likely to adopt this strategy and dermatologists the least likely $(51,88 \% \mathrm{v}$ $32,63 \%$ respectively, $\left.\chi^{2}=17 \cdot 0, \quad \mathrm{df}=7 ; \mathrm{p}<0.05\right)$. The principal drugs for which consultants wished to retain responsibility for monitoring varied with their specialty. Overall the drugs most commonly cited were immunosuppressants $(96(21 \%)$ consultants), cytotoxic drugs $(73(16 \%))$, and cardiovascular drugs $(64(14 \%))$. When consultants were asked which procedures might help to clarify who had clinical responsibility for outpatients the procedures they most commonly endorsed from a list of possibilities were the same as those given by general practitioners (table IV). There were no significant differences among the specialties in their responses.

Consultants often found it necessary to ask general practitioners to complete a short course of drug treatment initiated by the hospital: $171(38 \%)$ consultants made such requests at least weekly; 105 (23\%) did so at least once a month; and $44(10 \%)$ at least yearly. Gynaecologists were the most likely to do so and cardiologists the least likely (table V).

A total of $408(89 \%)$ consultants said that their hospital imposed limits on the quantities of drugs supplied to outpatients, and $319(70 \%)$ said that they were required to prescribe from a limited list. Consultants were asked which strategies from a list of possibilities they employed to circumvent these restrictions: $286(63 \%)$ asked the general practitioner to prescribe; $108(24 \%)$ insisted that the hospital pharmacy dispense as requested; $92(20 \%)$ used FP10(HP) prescription forms; and two $(2 \%)$ asked patients to return to the clinic more frequently. Gynaecologists were the most likely to ask general practitioners to prescribe whereas respiratory physicians were the least likely (table V).

Overall, $92(20 \%)$ consultants found it necessary to breach the hospital's dispensing policy weekly, 122 (27\%) monthly, and 109 (24\%) yearly. Dermatologists breached restrictions most frequently and rheumatologists least often (table V). The greater the hospital's restriction on the quantities of drugs supplied to outpatients, the more likely consultants were to avoid restrictions by asking the general practitioner to prescribe (table II).

\section{Discussion}

\section{LIMITATIONS OF STUDY}

Our findings need to be considered within the overall limitations of the study. The general practitioners and consultants selected for investigation are not representative of the country as a whole as they practised near or in one of 94 major acute hospitals, which in turn had been selected to include a disproportionate number of teaching hospitals and those with either very restrictive or very liberal outpatient dispensing policies. We assumed that general practitioners would refer most of their patients to these hospitals. However, they reported referring patients to a wide number of hospitals and did not respond to our questionnaire solely in relation to the selected hospital. Our data are likely, therefore, to underestimate the 
true strength of the associations between the selected hospital's outpatient dispensing policy and the general practitioners' views.

Consultants were drawn from eight medical specialties likely to experience prescribing problems in their interface with general practice and are not necessarily representative of consultants generally. In addition, as consultants in most hospitals were exempt from certain outpatient dispensing restrictions their experience does not reflect that of more junior medical staff, who receive no such exemption. An added limitation is that consultants were recruited to the study by hospital chief pharmacists, who may have preferentially selected those whom they thought would respond or whose views were similar to their own. This bias is offset by the fact that many hospitals have only one consultant in a given speciality, offering no scope for selection.

The response rates among general practitioners $(64 \%)$ and consultants $(63 \%)$ were not high, introducing the possibility of non-responder bias. Doctors with particular problems with hospital outpatient dispensing may be overrepresented in our sample. Nevertheless, if we were to assume that all non-responders had no problems with hospital outpatient dispensing policies the proportions who were dissatisfied would still be considerable. Among general practitioners there would remain a third who felt unable to take clinical responsibility, for certain drug treatments initiated by the hospital and half who had been asked at least monthly to complete a short course of treatment initiated by the hospital. Among consultants there would remain more than a third who found it necessary to circumvent the hospital's restrictions by asking the general practitioner to prescribe and half who wished to retain responsibility for prescribing certain drug treatments.

\section{IMPACT OF POLICY}

Nearly half of the general practitioners surveyed said that they had been asked by the hospital to prescribe drugs for which they felt unable to take clinical responsibility. General practitioners said that they lacked the knowledge and, in some instances, the technical resources needed to monitor drug dosage, side effects, and response to treatment. It could be argued that these aspects of patient care might be "shared" with the hospital doctor, who has the necessary expertise and resources. General practitioners, however, voiced strong objections to prescribing a drug treatment when they had no other clinical responsibility for the management of that aspect of the patient's condition. Consultants' views complemented those of general practitioners. Nearly three quarters of consultants wished to retain responsibility when there was a specialist need to initiate, stabilise, or monitor drug treatment.

Infertility drugs were the treatments with which general practitioners most often had difficulty. General practitioners volunteered that it was not appropriate that they should assume prescribing responsibility when treatment effects could not àdequately be monitored in general practice, particularly as they would share legal liability for possible adverse outcomes such as multiple pregnancy. Some hospitals have considered offering a legal waiver that would release general practitioners from the clinical responsibility of agreeing to prescribe certain specialist drugs. ${ }^{8}$ At present, however, there is no legal precedent for clinical responsibility to be dissociated from responsibility for prescribing. ${ }^{9}$

Improved communication between general practitioners and consultants was seen by both groups as a stratagem for ameliorating prescribing problems at the hospital-general practice interface. Most doctors in both groups endorsed the view that general practitioners should make clear in their letter of referral whether consultants should treat or only advise about treatment and that consultants should communicate promptly with general practitioners, explaining the proposed course of treatment. These suggestions amount to making an explicit agreement as to which doctor has clinical responsibility for which elements of the patient's care at any given time. If clinical responsibility carries with it the responsibility for prescribing such agreements would make clear who should write the drug prescription. Flexibility is essential as there was considerable variation among general practitioners in the drugs for which they felt unable to assume responsibility and among consultants in the drugs for which they wished to retain responsibility.

Restrictive hospital outpatient dispensing policies were seen as preventing general practitioners and consultants from implementing agreements which they would otherwise prefer. We found that the more restricted the supply of drugs given to outpatients the more frequently the consultant circumvented the restrictions by asking general practitioners to prescribe and the more likely general practitioners were to be asked to complete a short course of treatment initiated by the hospital. The respondents also volunteered that restrictive policies increased the workload of general practitioners, were inconvenient for patients, and added to overall NHS costs as many specialist drugs were more highly priced when dispensed in the community.

There was widespread concern that restrictions on the nature and quantities of drugs supplied to outpatients were intended mainly to save money, a strategy which served to shift clinical responsibility on to general practitioners, who were not always best able to assume such responsibility. General practitioners and consultants agreed that hospital doctors should be able to retain clinical responsibility, including that for prescribing, when they judged it was appropriate. If guidelines on prescribing at the hospital-general practice interface are to prove effective and beneficial they must address this central issue of which doctor is best able to assume clinical responsibility at any given time. Patients' views need to be sought about the implications of the provisions made for outpatient care in terms of their health, convenience, and personal cost.

We thank the hospital chief pharmacists, general practitioners, and hospital consultants who participated in the study, and our research staff Jenny Giles, Rosemary JambertGray, Matthew Macdonald, Linda MacRae-Samuel, Mary Taylor, Frances Smyth, Anne Woolhouse, and Lesley Woolhouse. The study was funded by a grant from the Department of Health.

1 NHS Management Board. Circular DA(87)10 to regional general managers, district general managers, and general managers of special health authorities for the
London postgraduate teaching hospitals. London: DHSS, 1987 . London postgraduate teaching hospitals. London: DHSS, 1987.

2 Beecham L. Transferring prescribing costs. BMf 1989;299:863.

3 Pennington S. Drugs cash carrot sparks FPC row. GP 1990 June 1:3.

4 Griffiths H. Handing out bitter pills. Pulse 1990 September 29:33.

Secretaries of State for Health, Wales, Northern Ireland, and Scotland. Promoting better health. London: HMSO, 1987. (Cm 249.)

6 Secretaries of State for Health, Wales, Northern Ireland, and Scotland. Working for patients. London: HMSO, 1989. (Cm 555 .

7 Wilkie P, Sibbald B, Freeling P, Raftery J, Anderson S. Prescribing at the hospital-general practice interface. I. Hospital outpatient dispensing policies in England. BMf 1992;304:29-31.

8 Wick A. Erythropoietin: legal waiver for GPs mooted by hospital. Pulse 199] June 8:3.

9 Crouch A. The medicolegal problems of transferred prescribing. Prescriber 1991 June 19:55-7.

(Accepted 25 September 1991) 